\title{
Embedding Theorems and Area Operators on Bergman Spaces with Doubling Measure
}

\author{
Changbao Pang ${ }^{1} \cdot$ Antti Perälä ${ }^{2}$ - Maofa Wang ${ }^{3}$
}

Received: 8 June 2020 / Accepted: 13 February 2021 / Published online: 3 March 2021

(c) The Author(s) 2021

\begin{abstract}
We establish an embedding theorem for the weighted Bergman spaces induced by a positive Borel measure $d \omega(y) d x$ with the doubling property $\omega(0,2 t) \leq C \omega(0, t)$. The characterization is given in terms of Carleson squares on the upper half-plane. As special cases, our result covers the standard weights and logarithmic weights. As an application, we also establish the boundedness of the area operator.
\end{abstract}

Keywords Weighted Bergman space $\cdot$ Carleson measure $\cdot$ Area operator

Mathematics Subject Classification Primary 47B35; Secondary 30H20

\section{Introduction}

Let $X$ be a space of functions on a domain $\Omega \subset \mathbb{C}$. A positive Borel measure $\mu$ on $\Omega$ is said to be a $p$-Carleson measure for $X$ if the embedding $X \subset L^{p}(\mu), 0<p<\infty$, is continuous. The problem of characterizing such measures for various choices of

Communicated by Eric Weber.

This work was partially supported by Natural Science Foundation of China (11771340).

凶 Antti Perälä

antti.perala@umu.se

Changbao Pang

cbpangmath@sxnu.edu.cn

Maofa Wang

mfwang.math@whu.edu.cn

1 School of Mathematics and Computer Science, Shanxi Normal University, Linfen 041000, China

2 Department of Mathematics and Mathematical Statistics, Umeå University, 90187 Umeå, Sweden

3 School of Mathematics and Statistics, Wuhan University, 430072 Wuhan, China

Birkhäuser 
$X$ is known as the Carleson embedding problem. These ideas originate from the pioneering work of Carleson [2,3], where they are motivated by interpolation and the corona problem. Since then, there has been a great amount of research on this topic, and this kind of measures have found many applications in other related areas [28].

It is natural to study the Carleson embedding problem in the context of various different spaces of functions. The problem on Hardy spaces over the half-plane $\Pi^{+}:=$ $\{z \in \mathbb{C}: \Im z>0\}$, on the $M$-harmonic Hardy spaces over the unit ball, and on the Bergman spaces over the unit disk equipped with a radial doubling weight have been resolved in $[1,15,19,20]$. The proofs employ techniques from harmonic analysis, such as the theory of tent spaces introduced by Coifman, Meyer and Stein [6] as well as Cohn and Verbitsky [5]. Carleson embedding theorems on Zen spaces satisfying a doubling condition have been characterized in [13], see also [14]. Following this line of reasoning, we obtain a description of the Carleson measures for a very general class of weighted Bergman spaces (or Zen spaces) on the upper half-plane $\Pi^{+}$.

To give the precise statement of our main result, we need to introduce some notation. Denote by $\Delta_{2}$ the class of positive Borel measures on $(0, \infty)$ satisfying

$$
0<\omega(0,2 t) \leq C \omega(0, t)<\infty
$$

for all $t \in(0, \infty)$, where $C>0$ is a constant. Let $H\left(\Pi^{+}\right)$denote the space of analytic functions on $\Pi^{+}$. For $0<p<\infty, \omega \in \Delta_{2}$, the weighted Bergman space $A_{\omega}^{p}\left(\Pi^{+}\right)$ consists of functions $f \in H\left(\Pi^{+}\right)$such that

$$
\|f\|_{A_{\omega}^{p}}^{p}:=\int_{\Pi^{+}}|f(z)|^{p} d(\omega \otimes m)(z)<\infty
$$

where $d(\omega \otimes m)(z)=d \omega(y) d x$ and $z=x+i y \in \Pi^{+}$. For simplicity, we also write $(f \omega)[E]=\int_{E} f d(\omega \otimes m)$ for each non-negative $f$. The $\Delta_{2}$ class and other classes of doubling weights have been studied extensively in the context of harmonic analysis and partial differential equations; see for instance [13,14,16,18,20-22,27].

We will frequently use the Poisson integral technique. Due to the unboundedness of $\Pi^{+}$, to recover $f$ from its boundary values on $\Pi_{a}^{+}:=\{z \in \mathbb{C}: \Im z>a\}$ for some $a>0$ via the Poisson integral, we always assume that $f \in H\left(\Pi^{+}\right)$is bounded on the closure of $\Pi_{a}^{+}$. Denote by $\mathbf{1}_{2}$ the subclass of $\omega \in \Delta_{2}$ for which is this true. For other measures in $\Delta_{2}$, we consider the spaces

$$
\mathcal{A}_{\omega}^{p}\left(\Pi^{+}\right):=\left\{f \in H\left(\Pi^{+}\right):\|f\|_{\mathcal{A}_{\omega}^{p}}^{p}:=\sup _{\epsilon>0} \int_{\Pi^{+}}|f(z+i \epsilon)|^{p} d(\omega \otimes m)(z)<\infty\right\} .
$$

Such spaces are also called Zen spaces [10,13,23]. It is known that $\mathcal{A}_{\omega}^{p}\left(\Pi^{+}\right) \subset$ $A_{\omega}^{p}\left(\Pi^{+}\right)$, each $f \in \mathcal{A}_{\omega}^{p}\left(\Pi^{+}\right)$is bounded on the closure of $\Pi_{a}^{+}$for any $a>0$ and satisfies $\|f\|_{\mathcal{A}_{\omega}^{p}}^{p}=\|f\|_{A_{\omega}^{p}}^{p}$. See [13] or Corollary 4.2 of [23].

Obviously, $A_{\omega}^{p}\left(\Pi^{+}\right)$is a Banach space for $\omega \in \mathbf{1}_{2}$ when $1 \leq p<\infty$, and a complete metric space, when $0<p<1$. If $d(\omega \otimes m)(z)=(\Im z)^{\alpha} d A(z)$, where $\alpha>-1$ and $d A$ is the area measure on $\Pi^{+}$, then we recover the standard weighted 
Bergman spaces $A_{\alpha}^{p}\left(\Pi^{+}\right)$. However, the class $\mathbf{1}_{2}$ is much larger than the class of the standard weights. For instance, let $\mathbf{1}_{2}^{\prime}$ be the class of continuous functions $\omega$ on $(0, \infty)$ satisfying

$$
\lim _{t \rightarrow 0^{+}} \omega(t)<\infty, \inf _{t \in(0, \infty)} \omega(t)>0
$$

and

$$
\lim _{t \rightarrow \infty} \frac{1}{t \omega(t)} \int_{0}^{t} \omega(y) d y>0
$$

Then $\omega(y) d y \in \mathbf{1}_{2}$ for every $\omega \in \mathbf{1}_{2}^{\prime}$ (see Remark 2.2 for more details). A typical example of $\omega \in \mathbf{1}_{2}^{\prime}$ is

$$
\omega(t)= \begin{cases}\ln t, & \text { if } t>e, \\ 1, & \text { if } 0<t \leq e .\end{cases}
$$

The class $\mathbf{1}_{2}^{\prime}$ can be compared with the class of regular weights, defined on the unit disk in terms of a distortion function by Siskakis, see for instance $[19,26]$.

It is easily seen that each point evaluation $L_{z}$ induced by $z \in \Pi^{+}$is a bounded linear functional on $A_{\omega}^{2}\left(\Pi^{+}\right), \omega \in \mathbf{1}_{2}$ (see Sect. 3). Therefore, according to the Riesz representation theorem, there exist reproducing kernels $B_{z}^{\omega} \in A_{\omega}^{2}\left(\Pi^{+}\right)$with $\left\|L_{z}\right\|=$ $\left\|B_{z}^{\omega}\right\|_{A_{\omega}^{2}}$ having the reproducing property

$$
f(z)=\int_{\Pi^{+}} f(\zeta) \overline{B_{z}^{\omega}(\zeta)} d(\omega \otimes m)(\zeta), \quad f \in A_{\omega}^{2}\left(\Pi^{+}\right) .
$$

Nevertheless, many other basic properties of the Bergman spaces $A_{\omega}^{p}\left(\Pi^{+}\right), \omega \in \mathbf{1}_{2}$, are not yet well understood and have indeed attracted more attention in the recent years. The theory for such spaces is both interesting and involving, partly because the usual techniques for the standard Bergman spaces fail to work. For example, we do not know whether the natural Bergman projection

$$
P_{\omega}(f)(z)=\int_{\Pi^{+}} f(\zeta) \overline{B_{z}^{\omega}(\zeta)} d(\omega \otimes m)(\zeta), \quad z \in \Pi^{+},
$$

is bounded on $L_{\omega}^{p}\left(\Pi^{+}\right), p \neq 2$. This, in part, explains why the dual spaces of $A_{\omega}^{p}\left(\Pi^{+}\right)$ have not been identified. It is worth noting that the corresponding result for the unit disk is now settled in the affirmative in the recent remarkable paper [21]. However, it is not obvious whether their method carries over to the setting of the present paper.

The purpose of this work is to give the embedding theorem for weighted Bergman spaces $A_{\omega}^{p}\left(\Pi^{+}\right)$. Our main tools are the admissible maximal function and an adaptation of the stopping time argument in [6], which was obtained for this type of spaces on the disk by Peláez and Rättyä [20]. To this end, we need to introduce some further notation. For an interval $I \subset \mathbb{R}(\mathbb{R}$ is the real axis on $\mathbb{C})$, let

$$
Q_{I}=\left\{z=x+i y \in \Pi^{+}: x \in I, 0<y \leq|I|\right\}
$$


denote the Carleson square, where $|I|$ stands for the length of the interval $I$. It is also convenient to define the intervals

$$
I_{z}=\left\{x \in \mathbb{R}:|\Re z-x| \leq \frac{1}{2} \Im z\right\}
$$

for each $z \in \Pi^{+}$and to denote $Q(z)=Q_{I_{z}}$. For $\xi \in \Pi^{+}$, we define the truncated cone

$$
\Lambda_{T}(\xi)=\left\{z \in \Pi^{+}:|\Re z-\Re \xi|<\frac{1}{2}(\Im z-\Im \xi)\right\} .
$$

The tent related to $\Lambda_{T}(\xi)$ is given by

$$
T(z)=\left\{\xi \in \Pi^{+}: z \in \Lambda_{T}(\xi)\right\}
$$

Finally, we define the admissible maximal function over the truncated cone $\Lambda_{T}(z)$ by

$$
N_{T} f(z)=\sup _{w \in \Lambda_{T}(z)}|f(w)|, \quad z \in \Pi^{+} .
$$

Throughout the paper we use the same letter $C$ to denote various positive constants which may change at each occurrence. Variables indicating the dependency of constants $C$ will be often specified in parenthesis. We use the notation $X \lesssim Y$ or $Y \gtrsim X$ for non-negative quantities $X$ and $Y$ to mean $X \leq C Y$ for some inessential constant $C>0$. Similarly, we use the notation $X \approx Y$ if both $X \lesssim Y$ and $Y \lesssim X$ hold. Given $p \in[1, \infty]$, we will denote by $p^{\prime}=p /(p-1)$ its Hölder conjugate. In this context we agree that $1^{\prime}=\infty$ and $\infty^{\prime}=1$.

Our first result characterizes the $p$-Carleson measures for $A_{\omega}^{p}\left(\Pi^{+}\right)$.

Theorem 1.1 Let $0<p<\infty, \omega \in \mathbf{1}_{2}$, and let $\mu$ be a positive Borel measure on $\Pi^{+}$. Then the following statements are equivalent:

(1) $\mu$ is a p-Carleson measure for $A_{\omega}^{p}\left(\Pi^{+}\right)$.

$$
\sup _{z \in \Pi^{+}} \frac{\mu[Q(z)]}{\omega[Q(z)]}<\infty
$$

(3) There exists a constant $\eta_{0}=\eta_{0}(\omega)>1$ such that for all $\eta>\eta_{0}$,

$$
\sup _{z \in \Pi^{+}} \frac{1}{\omega[Q(z)]} \int_{\Pi^{+}}\left(\frac{\Im z}{|w-\bar{z}|}\right)^{\eta} d \mu(w)<\infty .
$$

Moreover, if $\mu$ is a p-Carleson measure for $A_{\omega}^{p}\left(\Pi^{+}\right)$, then the norm of the identity mapping $I_{d}: A_{\omega}^{p}\left(\Pi^{+}\right) \rightarrow L^{p}(\mu)$ satisfies

$$
\left\|I_{d}\right\|^{p} \approx \sup _{z \in \Pi^{+}} \frac{\mu[Q(z)]}{\omega[Q(z)]} \approx \sup _{z \in \Pi^{+}} \frac{1}{\omega[Q(z)]} \int_{\Pi^{+}}\left(\frac{\Im z}{|w-\bar{z}|}\right)^{\eta} d \mu(w) .
$$


Theorem 1.1 for $p \geq 1$ is essentially covered in [13]. Here we present an alternative proof, which covers $0<p<1$, using ideas from [19,20].

Put, $\widehat{\Pi^{+}}=\overline{\Pi^{+}} \cup\{\infty\}$. We say $\lim _{z \rightarrow \partial \widehat{\Pi^{+}}} g(z)=0$, if $g(z) \rightarrow 0$ as $\Im z \rightarrow 0^{+}$or $|z| \rightarrow \infty$. This is equivalent to saying that for every $\varepsilon>0$, there is a compact set $K \subset \Pi^{+}$such that $\sup _{z \in \Pi^{+} \backslash K}|g(z)|<\varepsilon$.

Assume that $\left\{f_{n}\right\}$ is a bounded sequence of functions in $A_{\omega}^{p}\left(\Pi^{+}\right)$and $f_{n} \rightarrow 0$ uniformly on compact subsets of $\Pi^{+}$as $n \rightarrow \infty$. A positive measure $\mu$ is called a compact $p$-Carleson measure (or a vanishing Carleson measure, see [28]), if the $L^{p}(\mu)$ norms of $f_{n}$ tend to 0 as $n \rightarrow \infty$. The following theorem characterizes the compact $p$-Carleson measures for $A_{\omega}^{p}\left(\Pi^{+}\right)$.

Theorem 1.2 Let $0<p<\infty, \omega \in \mathbf{1}_{2}$, and let $\mu$ be a positive Borel measure on $\Pi^{+}$, which is finite on any compact subset of $\Pi^{+}$. Then the following statements are equivalent:

(1) $\mu$ is a compact p-Carleson measure for $A_{\omega}^{p}\left(\Pi^{+}\right)$.

(2)

$$
\lim _{z \rightarrow \frac{\partial \Pi^{+}}{\omega[Q(z)]}} \frac{\mu[Q(z)]}{\omega[}
$$

(3) There exists a constant $\eta_{0}=\eta_{0}(\omega)>1$ such that for all $\eta>\eta_{0}$,

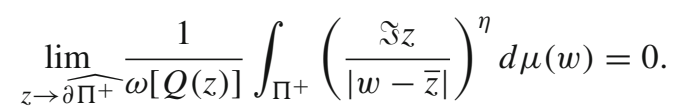

We note that in both Theorems 1.1 and 1.2 the condition of being a (resp. compact) $p$-Carleson measure for $A_{\omega}^{p}\left(\Pi^{+}\right)$is invariant on $p$; and we could very well just call these measures (resp. compact) Carleson measures. See [19,20] for a detailed study of $p$-Carleson measures for $q$-integrable Bergman spaces on the disk. Embedding theorems for derivatives of Hardy spaces were obtained in [15], and for doubling Bergman spaces of the unit disk, we refer to [20]. As for compact Carleson measures, similar results and methodology can be found in [17] for Bergman spaces induced by rapidly decreasing weights, and in [19] for Bergman spaces induced by rapidly increasing weights; see also [22] for doubling weights.

As an application of the Carleson embedding theorems, the boundedness of certain area operators can be obtained. We recall that for $0<s<\infty$, the generalized area operator induced by a positive Borel measure $\mu$ on $\Pi^{+}$is defined by

$$
A_{s}^{\mu} f(z)=\left(\int_{\Lambda_{T}(z)}|f(w)|^{s} d \mu(w)\right)^{\frac{1}{s}}, \quad z \in \Pi^{+} .
$$

It is worth mentioning that the area operator is tremendously useful in harmonic analysis. It is, for example, related to the Littlewood-Paley operator, multipliers and tent spaces. The boundedness of area operators on Hardy spaces, Hardy-Sobolev spaces and Bergman spaces on the unit disk has been considered in [4,9]. Similar problems 
and ideas for weighted Bergman spaces on the disk, where the weight satisfies a doubling property, have been previously studied in [22] (where the problem is solved for more general parameters). We employ admissible (non-tangential) maximal functions and analysis of maximal operators on a dyadic grid (see [12,22,24]) to study the boundedness of area operator $A_{s}^{\mu}$ on $A_{\omega}^{p}\left(\Pi^{+}\right)$.

The following result characterizes the boundedness of the area operator $A_{s}^{\mu}$.

Theorem 1.3 Let $0<s, p<\infty$ and $\omega \in \mathbf{1}_{2}$. Let $\mu$ be a positive Borel measure on $\Pi^{+}$which is finite on compact subsets of $\Pi^{+}$. Assume that the area operator $A_{s}^{\mu}$ is well-defined on $A_{\omega}^{p}\left(\Pi^{+}\right)$. Then $A_{s}^{\mu}: A_{\omega}^{p}\left(\Pi^{+}\right) \rightarrow L^{p}(d(\omega \otimes m))$ is bounded if and only if

$$
d \mu_{\omega}(z):=\omega[T(z)] d \mu(z)
$$

is a p-Carleson measure for $A_{\omega}^{p}\left(\Pi^{+}\right)$. Moreover, in this case,

$$
\left\|A_{s}^{\mu}\right\|_{\left(A_{\omega}^{p}, L^{p}(d(\omega \otimes m))\right)}^{s} \approx \sup _{z \in \Pi^{+}} \frac{\mu_{\omega}[Q(z)]}{\omega[Q(z)]} .
$$

As mentioned before, the results in Theorems 1.1,1.2, and 1.3 hold true for $\mathcal{A}_{\omega}^{p}\left(\Pi^{+}\right)$ when $\omega \in \Delta_{2}$ with the same proofs.

After collecting some preliminaries in Sect. 2, we prove our main Theorems 1.1 and 1.2 in Sect. 3 and Theorem 1.3 in Sect. 4.

\section{Preliminaries}

In this section we present some preliminary results about the weights $\omega \in \Delta_{2}$. These will be frequently used in the sequel. In the setting of the unit disk, there exists a great number of results and characterizations for doubling weights, see [18-20]. The following result is analogous to Lemma 1 in [20] (see also Lemma 2.1 of [18]).

Lemma 2.1 The following statements hold.

(1) $\omega \in \Delta_{2}$ if and only if there exist $C=C(\omega) \geq 1$ and $\beta=\beta(\omega)>0$ such that

$$
\int_{0}^{s} d \omega(y) \leq C\left(\frac{s}{t}\right)^{\beta} \int_{0}^{t} d \omega(y), \quad 0<t \leq s<\infty .
$$

(2) Let $0<p<\infty$ and $\omega \in \Delta_{2}$. There exists a constant $\lambda_{0}=\lambda_{0}(\omega)>0$ such that for all $\lambda>\lambda_{0}$ and each $a \in \Pi^{+}$, the function

$$
F_{a, p}(z)=\left(\frac{\Im a}{z-\bar{a}}\right)^{\frac{\lambda+1}{p}}
$$

is analytic in $\Pi^{+}$and satisfies

$$
\left|F_{a, p}(z)\right| \approx 1, \quad z \in Q(a)
$$


and

$$
\left\|F_{a, p}\right\|_{A_{\omega}^{p}}^{p} \approx \omega[Q(a)]
$$

Proof For (1), let $\omega \in \Delta_{2}$ and $0<t \leq s<\infty$. Let $s_{n}=2^{n}$ for all $n \in \mathbb{Z}$. Then there exist $k \in \mathbb{Z}$ and $m \in \mathbb{Z}$ with $m \leq k$ such that $s_{k} \leq s<s_{k+1}$ and $s_{m} \leq t<s_{m+1}$. By the doubling property (1.1), there exists $C=C(\omega)>0$ such that

$$
\begin{aligned}
\int_{0}^{s} d \omega(y) & \leq \int_{0}^{s_{k+1}} d \omega(y) \leq C \int_{0}^{s_{k}} d \omega(y) \\
& \leq \cdots \leq C^{k-m+1} \int_{0}^{s_{m}} d \omega(y) \\
& \leq C^{k-m+1} \int_{0}^{t} d \omega(y) \\
& =C^{2} 2^{(k-m-1) \log _{2} C} \int_{0}^{t} d \omega(y) \\
& \leq C^{2}\left(\frac{s}{t}\right)^{\log _{2} C} \int_{0}^{t} d \omega(y)
\end{aligned}
$$

Conversely, the choice $t=s / 2$ in (2.1) gives $\omega \in \Delta_{2}$.

For (2), let $\omega \in \Delta_{2}$ and $\lambda>\beta+1$, where $\beta=\beta(\omega)$ is the constant in (1). For $k \in \mathbb{N} \cup\{0\}$ and $z \in \Pi^{+}$, denote

$$
Q_{k}(z)=\left\{w \in \Pi^{+}:|\Re z-\Re w|<2^{k-1} \mathfrak{\Im} z, \Im w \leq 2^{k} \Im z\right\}
$$

Then

$$
\frac{2^{k}}{2} \Im z \leq|\Re w-\Re z| \leq \sqrt{|\Re w-\Re z|^{2}+(\Im w+\Im z)^{2}}=|w-\bar{z}|,
$$

if $w \in\left(Q_{k+1}(z) \backslash Q_{k}(z)\right) \cap\left\{w \in \Pi^{+}: \Im w \leq 2^{k} \Im z\right\}, k \in \mathbb{N} \cup\{0\}$. Also,

$$
\frac{2^{k}}{2} \Im z<\Im w \leq|w-\bar{z}|
$$

if $w \in\left(Q_{k+1}(z) \backslash Q_{k}(z)\right) \cap\left\{w \in \Pi^{+}: \Im w>2^{k} \Im z\right\}, k \in \mathbb{N} \cup\{0\}$. Moreover,

$$
0<\mathfrak{\Im} z \leq|w-\bar{z}|,
$$


if $w \in Q_{0}(z)$. Thus,

$$
\begin{aligned}
\int_{\Pi^{+}} \frac{1}{|w-\bar{z}|^{\lambda+1}} d(\omega \otimes m)(w)= & \int_{Q_{0}(z)} \frac{1}{|w-\bar{z}|^{\lambda+1}} d(\omega \otimes m)(w) \\
& +\sum_{k=0}^{\infty} \int_{Q_{k+1}(z) \backslash Q_{k}(z)} \frac{1}{|w-\bar{z}|^{\lambda+1}} d(\omega \otimes m)(w) \\
& \lesssim \sum_{k=0}^{\infty} \frac{1}{2^{k(\lambda+1)}(\Im z)^{\lambda+1}} \int_{Q_{k}(z)} d(\omega \otimes m)(w) \\
= & \sum_{k=0}^{\infty} \frac{2^{k} \Im z}{2^{k(\lambda+1)}(\Im z)^{\lambda+1}} \int_{0}^{2^{k} \Im z} d \omega(y) .
\end{aligned}
$$

Due to (2.1), there exists a constant $C=C(\omega)>0$ such that

$$
\int_{0}^{2^{k} \Im z} d \omega(y) \leq C 2^{k \beta} \int_{0}^{\Im z} d \omega(y) .
$$

Thus,

$$
\begin{aligned}
\int_{\Pi^{+}} \frac{1}{|w-\bar{z}|^{\lambda+1}} d(\omega \otimes m)(w) & \lesssim \sum_{k=0}^{\infty} \frac{1}{2^{k(\lambda-\beta)}(\Im z)^{\lambda}} \int_{0}^{\Im z} d \omega(y) \\
& \lesssim \frac{1}{(\Im z)^{\lambda}} \int_{0}^{\Im z} d \omega(y) .
\end{aligned}
$$

On the other hand,

$$
\begin{aligned}
\int_{\Pi^{+}} \frac{1}{|w-\bar{z}|^{\lambda+1}} d(\omega \otimes m)(w) & \gtrsim \int_{Q(z)} \frac{1}{|w-\bar{z}|^{\lambda+1}} d(\omega \otimes m)(w) \\
& \gtrsim \frac{1}{(\Im z)^{\lambda+1}} \omega[Q(z)] \\
& =\frac{1}{(\Im z)^{\lambda}} \int_{0}^{\Im z} d \omega(y) .
\end{aligned}
$$

This completes the proof of (2.3). A straightforward calculation gives (2.2).

Remark 2.2 As promised in the introduction, we show that $\omega(y) d y \in \mathbf{1}_{2}$ for all $\omega \in \mathbf{1}_{2}^{\prime}$. In fact, assume that

$$
\lim _{t \rightarrow \infty} \frac{1}{t \omega(t)} \int_{0}^{t} \omega(y) d y=C>0 .
$$

A direct calculation shows that there exists $t_{0} \in(0, \infty)$ such that

$$
\frac{1}{2} C t \omega(t)<\int_{0}^{t} \omega(y) d y
$$


for all $t \in\left[t_{0}, \infty\right)$. Then, a differentiation yields that the function

$$
h(t):=\frac{1}{t \frac{2}{C}} \int_{0}^{t} \omega(y) d y
$$

is decreasing on $\left[t_{0}, \infty\right)$. Therefore,

$$
\int_{0}^{t} \omega(y) d y \leq 2^{\frac{2}{C}} \int_{0}^{\frac{t}{2}} \omega(y) d y
$$

for $t \in\left[2 t_{0}, \infty\right)$. For $t \in\left(0,2 t_{0}\right]$,

$$
\int_{0}^{t} \omega(y) d y \leq \frac{2 \sup _{t \in\left[0, t_{0}\right]} \omega(t)}{\inf _{t \in\left(0,2 t_{0}\right]} \omega(t)} \int_{0}^{\frac{t}{2}} \omega(y) d y
$$

By (2.6) and (2.7), $\omega(y) d y \in \mathbf{1}_{2}$. The standard estimate obtained from the mean-value property for analytic functions shows that if $f \in A_{\omega}^{p}\left(\Pi^{+}\right)$, then

$$
|f(z)|^{p} \lesssim \frac{1}{(\Im z)^{2}} \int_{\Pi^{+}}|f(w)|^{p} d A(w) \lesssim \frac{1}{\Im z^{2}} \int_{0}^{\infty} \int_{-\infty}^{\infty}|f(x+i y)|^{p} d x \omega(y) d y .
$$

Therefore, $f$ is bounded on $\Pi_{a}^{+}$for any $a>0$ if $f \in H\left(\Pi^{+}\right)$and

$$
\int_{0}^{\infty} \int_{-\infty}^{\infty}|f(x+i y)|^{p} d x \omega(y) d y<\infty .
$$

\section{Carleson Embedding Theorem}

This section is devoted to the proofs of Theorems 1.1 and 1.2. To this end, we recall some facts about the Hardy spaces $H^{p}$ over the half-plane. For $0<p<\infty$, we say $f \in H^{p}$ if $f \in H\left(\Pi^{+}\right)$and

$$
\|f\|_{H^{p}}^{p}:=\sup _{y>0} \int_{-\infty}^{\infty}|f(x+i y)|^{p} d x<\infty .
$$

For $f \in H^{p}$, it is well-known ([7, P. 189, P.191]) that the boundary function $f(x):=$ $\lim _{y \rightarrow 0} f(x+i y)$ exists almost everywhere and

$$
\sup _{y>0} \int_{-\infty}^{\infty}|f(x+i y)|^{p} d x=\int_{-\infty}^{\infty}|f(x)|^{p} d x .
$$

We will need the $L^{p}$ boundedness of the non-tangential maximal function. The idea is to reduce to the Hardy space case as in [19]. The following fact is immediate from the definition of our spaces. 
Lemma 3.1 Let $0<p<\infty$, and $\omega \in \mathbf{1}_{2}$. Put $f_{t}(z)=f(z+$ it $)$ where $t \in(0, \infty)$ and $z \in \Pi^{+}$. If $f \in A_{\omega}^{p}\left(\Pi^{+}\right)$, then $f_{t} \in H^{p}$ for each $t>0$.

We proceed to the proof regarding the non-tangential maximal function.

Lemma 3.2 Let $0<p<\infty$ and $\omega \in \mathbf{1}_{2}$. Then there exists a constant $C>0$ such that

$$
\left\|N_{T} f\right\|_{L^{p}(d(\omega \otimes m))}^{p} \leq C\|f\|_{A_{\omega}^{p}}^{p}
$$

for all $f \in A_{\omega}^{p}\left(\Pi^{+}\right)$.

Proof Put $f_{y}(z)=f(z+i y)$ where $y \in(0, \infty), z \in \Pi^{+}$and $f \in A_{\omega}^{p}\left(\Pi^{+}\right)$. For $x \in \mathbb{R}$, we denote by $\Gamma(x)$ the standard cone whose vertex is $x \in \mathbb{R}$, i.e.,

$$
\Gamma(x)=\left\{z \in \Pi^{+}:|\Re z-x|<\Im z\right\} .
$$

Define the non-tangential maximal function over the standard cone by

$$
f_{y}^{*}(x)=\sup _{w \in \Gamma(x)}\left|f_{y}(w)\right|
$$

Then

$$
f_{y}^{*}(x)=\sup _{w \in \Gamma(x)}\left|f_{y}(w)\right| \geq N_{T} f(z), \quad z=x+i y .
$$

For $f \in A_{\omega}^{p}\left(\Pi^{+}\right)$, by (3.1), Lemma 3.1 and [8, Theorem 3.1], we deduce that there exists a constant $C>0$ such that

$$
\begin{aligned}
\int_{\Pi^{+}}\left(N_{T} f(z)\right)^{p} d(\omega \otimes m)(z) & \leq \int_{0}^{\infty} \omega(y) \int_{-\infty}^{\infty}\left(f_{y}^{*}(x)\right)^{p} d x d y \\
& \leq C \int_{0}^{\infty} \omega(y) \int_{-\infty}^{\infty}\left|f_{y}(x)\right|^{p} d x d y \\
& \lesssim\|f\|_{A_{\omega}^{p}}^{p},
\end{aligned}
$$

which gives the desired result.

For $z \in \Pi^{+}$and $h \in(0, \infty]$, we define $\Lambda_{T}^{h}(z)$ as follows:

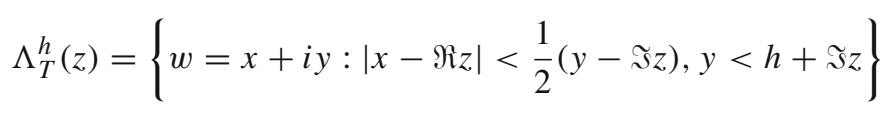

and set

$$
A_{p, \mu}^{p}(g \mid h)(\xi)=\int_{\Lambda_{T}^{h}(\xi)}|g(z)|^{p} d \mu(z)
$$


for a $\mu$-measurable function $g$. Note that $A_{p, \mu}^{p}(g \mid h)(\xi)$ is non-decreasing as a function of $h$.

For $0<p<\infty$ and a measurable function $f$ on $\Pi^{+}$, we define

$$
C_{p, \mu}^{p}(f)(z)=\sup _{a \in \Lambda_{T}(z)} \frac{1}{\omega[T(a)]} \int_{T(a)}|f(w)|^{p} \omega[T(w)] d \mu(w) .
$$

The following lemma is an essential part for establishing the duality of various tent spaces, see $[6,20]$. Since we forgo further discussion on tent spaces, we only present this result as an estimate.

Lemma 3.3 Let $\omega \in \Delta_{2}$ and $\mu$ be a positive Borel measure on $\Pi^{+}$. For any measurable function $g$ on $\Pi^{+}$, if $\sup _{z \in \Pi^{+}} C_{p, \mu}(g)(z)<\infty$, then there exist constants $M=$ $M(\omega)>0$ and $C=C(\omega)>0$ such that the function

$$
h(\xi):=\sup \left\{h: A_{p, \mu}(g \mid h)(\xi) \leq M C_{p, \mu}(g)(\xi)\right\}
$$

satisfies

$$
\int_{\Pi^{+}} k(z) \omega[T(z)] d \mu(z) \leq C \int_{\Pi^{+}}\left(\int_{\Lambda_{T}^{h(\xi)}(\xi)} k(z) d \mu(z)\right) d(\omega \otimes m)(\xi)
$$

for all $\mu$-measurable non-negative functions $k$.

Proof It follows from Fubini's theorem that

$$
\begin{aligned}
& \int_{\Pi^{+}}\left(\int_{\Lambda_{T}^{h(\xi)}(\xi)} k(z) d \mu(z)\right) d(\omega \otimes m)(\xi) \\
& =\int_{\Pi^{+}}\left(\int_{T(z) \cap H(z)} d(\omega \otimes m)(\xi)\right) k(z) d \mu(z),
\end{aligned}
$$

where $H(z)=\left\{\xi \in \Pi^{+}: \Im z-h(\xi)<\Im \xi<\Im z\right\}$. It suffices to show that

$$
\frac{1}{\omega[T(z)]} \int_{T(z) \cap H(z)} d(\omega \otimes m)(\xi) \geq C
$$

for all $z \in \Pi^{+}$. For $z \in \Pi^{+}$and $u \in \Pi^{+}$, we require $\Im u<3 \Im z$ to ensure that

$$
G(z, u):=T(z) \cap T(u) \cap\left\{\xi \in \Pi^{+}: \Im u-2 \Im z<\Im \xi\right\} \neq \emptyset .
$$


Let $z^{\prime}=\Re z+i 8 \Im z$. We deduce from Fubini's theorem and (2.1) that

$$
\begin{aligned}
& \frac{1}{\omega[T(z)]} \int_{T(z)} A_{p, \mu}^{p}(g \mid 2 \Im z)(\xi) d A_{\omega}(\xi) \\
& =\frac{1}{\omega[T(z)]} \int_{T(z)}\left(\int_{\Lambda_{T}^{2 \Im z}(\xi)}|g(u)|^{p} d \mu(u)\right) d(\omega \otimes m)(\xi) \\
& \quad=\frac{1}{\omega[T(z)]} \int_{\Pi^{+}}\left(\int_{G(z, u)} \omega(\xi) d A(\xi)\right)|g(u)|^{p} d \mu(u) \\
& \leq \frac{1}{\omega[T(z)]} \int_{T\left(z^{\prime}\right)}\left(\int_{T(u)} \omega(\xi) d A(\xi)\right)|g(u)|^{p} d \mu(u) \\
& \leq C_{1} \frac{1}{\omega\left[T\left(z^{\prime}\right)\right]} \int_{T\left(z^{\prime}\right)}^{|g(u)|^{p} \omega[T(u)] d \mu(u)} \\
& \leq C_{1} \inf _{v \in T(z)} C_{p, \mu}^{p}(g)(v),
\end{aligned}
$$

where the last inequality is valid due to

$$
\begin{aligned}
& \frac{1}{\omega\left[T\left(z^{\prime}\right)\right]} \int_{T\left(z^{\prime}\right)}|g(u)|^{p} \omega[T(u)] d \mu(u) \\
& \leq \sup _{a \in \Lambda_{T}(v)} \frac{1}{\omega[T(a)]} \int_{T(a)}|g(u)|^{p} \omega[T(u)] d \mu(u)
\end{aligned}
$$

for all $v \in T(z)$. Denote

$$
E(z)=\left\{\xi \in \Pi^{+}: \Im \xi \leq \Im z-h(\xi)\right\}
$$

Choosing $M$ so that $M^{p}>2 C_{1}$, by the definition of $h(\xi)$ and (3.3), we deduce that

$$
\begin{aligned}
& \frac{\omega[T(z) \cap H(z)]}{\omega[T(z)]}=1-\frac{\omega[T(z) \cap E(z)]}{\omega[T(z)]} \\
& \geq 1-\frac{1}{M^{p} \omega[T(z)] \inf _{v \in T(z)} C_{p, \mu}^{p}(g)(v)} \int_{T(z) \cap E(z)} A_{p, \mu}^{p}(g \mid 2 \Im z)(\xi) d(\omega \otimes m)(\xi) \\
& \geq 1-\frac{C_{1}}{M^{p}} \geq \frac{1}{2}
\end{aligned}
$$

which completes the proof.

We are now ready to prove the Carleson embedding theorem.

Proof of Theorem 1.1 For the implication (1) $\Rightarrow(3)$, assume that $\mu$ is a $p$-Carleson measure for $A_{\omega}^{p}\left(\Pi^{+}\right)$. Considering the test functions

$$
F_{z, p}(w)=\left(\frac{\Im z}{w-\bar{z}}\right)^{\frac{\eta}{p}}, \quad \eta>\lambda_{0}+1,
$$


defined in Lemma 2.1, we have

$$
\int_{\Pi^{+}}\left|F_{z, p}(w)\right|^{p} d \mu(w) \lesssim\left\|F_{z, p}\right\|_{A_{\omega}^{p}}^{p} \lesssim \omega[Q(z)]
$$

for all $z \in \Pi^{+}$, which implies (3) and

$$
\left\|I_{d}\right\|^{p} \gtrsim \sup _{z \in \Pi^{+}} \frac{1}{\omega[Q(z)]} \int_{\Pi^{+}}\left(\frac{\Im z}{|w-\bar{z}|}\right)^{\eta} d \mu(w) .
$$

The implication (3) $\Rightarrow(2)$ is immediate. We now prove $(2) \Rightarrow(1)$ by adapting the estimate from [5, p. 313] or [20, p. 215]. Suppose

$$
\sup _{z \in \Pi^{+}} \frac{\mu[Q(z)]}{\omega[Q(z)]}<\infty \text {. }
$$

Let $g(w)=\left(\frac{1}{\omega[T(w)]}\right)^{\frac{1}{p}}$, then

$$
\sup _{z \in \Pi^{+}} C_{p, \mu}(g)(z) \lesssim \sup _{z \in \Pi^{+}} \frac{\mu[Q(z)]}{\omega[Q(z)]}<\infty .
$$

For $f \in A_{\omega}^{p}\left(\Pi^{+}\right)$, using Lemma 3.3 with $k(z)=|f(z) g(z)|^{p}$, we deduce from Lemma 3.2 that

$$
\begin{aligned}
\int_{\Pi^{+}}|f(z)|^{p} d \mu(z) & \leq \int_{\Pi^{+}} \int_{\Lambda_{T}^{h(\xi)}(\xi)}|f(z) g(z)|^{p} d \mu(z) d(\omega \otimes m)(\xi) \\
& \leq \int_{\Pi^{+}}\left(N_{T} f(\xi)\right)^{p} \int_{\Lambda_{T}^{h(\xi)}(\xi)}(g(z))^{p} d \mu(z) d(\omega \otimes m)(\xi) \\
& \lesssim \int_{\Pi^{+}}\left(N_{T} f(\xi)\right)^{p} C_{p, \mu}(g)(\xi) d(\omega \otimes m)(\xi) \\
& \leq\|f\|_{A_{\omega}^{p}}^{p} \sup _{\xi \in \Pi^{+}} \frac{\mu[Q(\xi)]}{\omega[Q(\xi)]}
\end{aligned}
$$

where

$$
h(\xi)=\sup \left\{h: A_{p, \mu}(g \mid h)(\xi) \leq M C_{p, \mu}(g)(\xi)\right\}
$$

This calculation implies that $\mu$ is a $p$-Carleson measure for $A_{\omega}^{p}\left(\Pi^{+}\right)$and the norm of $I_{d}: A_{\omega}^{p}\left(\Pi^{+}\right) \rightarrow L^{p}(\mu)$ satisfies

$$
\left\|I_{d}\right\|^{p} \lesssim \sup _{z \in \Pi^{+}} \frac{\mu[Q(z)]}{\omega[Q(z)]}
$$


We define the weighted Hörmander type maximal function [11] as follows

$$
M_{\omega}(\varphi)(z)=\sup _{I: z \in Q_{I}} \frac{1}{\omega\left[Q_{I}\right]} \int_{Q_{I}}|\varphi(w)| d(\omega \otimes m)(w), \quad z \in \Pi^{+} .
$$

The role on $A_{\omega}^{p}\left(\Pi^{+}\right)$played by this maximal function is similar to that on $H^{p}$ played by the Hardy-Littlewood maximal function. A variant of the following lemma can be found in [11], and it can found for doubling weights on the disk in [18] (see also [19]). The lemma is of independent interest, and will be used in the proof of compact Carleson embedding theorem. Therefore, we present here a proof in the setting of the present paper.

Lemma 3.4 Let $0<p, q<\infty$ and $\omega \in \mathbf{1}_{2}$. Then there exists a constant $C=$ $C(p, \omega)>0$ such that

$$
|f(z)|^{p} \leq C M_{\omega}\left(f^{p}\right)(z), \quad z \in \Pi^{+},
$$

for all $f \in A_{\omega}^{q}\left(\Pi^{+}\right)$.

Proof Let $\omega \in \mathbf{1}_{2}$ and $\beta=\beta(\omega)>0$ be the constant in Lemma 2.1. Write $p=\alpha \gamma$, where $\gamma>\beta+1$. Let $\gamma^{\prime}=\frac{\gamma}{\gamma-1}$ and $0<t<\frac{\Im z}{2}$ for $z \in \Pi^{+}$. For $f \in A_{\omega}^{q}\left(\Pi^{+}\right)$, using the subharmonicity of $|f|^{\alpha}$ and Hölder's inequality, we have

$$
\begin{aligned}
|f(z)|^{\alpha} \leq & \frac{1}{\pi} \int_{-\infty}^{\infty} \frac{\Im z-t}{|z-i t-s|^{2}}|f(s+i t)|^{\alpha} d s \\
\leq & \left(\frac{1}{\pi} \int_{-\infty}^{\infty} \frac{(\Im z-t)^{\gamma-1}}{|z-i t-s|^{\gamma}}|f(s+i t)|^{\alpha \gamma} d s\right)^{\frac{1}{\gamma}} \\
& \times\left(\frac{1}{\pi} \int_{-\infty}^{\infty} \frac{(\Im z-t)^{\gamma^{\prime}-1}}{|z-i t-s|^{\gamma^{\prime}}} d s\right)^{\frac{1}{\gamma^{\prime}}} .
\end{aligned}
$$

That is,

$$
\begin{aligned}
|f(z)|^{p} & \leq C(\omega, p)\left(\frac{1}{\pi} \int_{-\infty}^{\infty} \frac{(\Im z-t)^{\gamma-1}}{|z-i t-s|^{\gamma}}|f(s+i t)|^{\alpha \gamma} d s\right) \\
& =C(\omega, p) \int_{-\infty}^{\infty} P_{\gamma}(z-t, s)|f(s+i t)|^{p} d s
\end{aligned}
$$

where

$$
P_{\gamma}(z-t, s)=\frac{1}{\pi} \frac{(\Im z-t)^{\gamma-1}}{|z-i t-s|^{\gamma}}
$$


Set $s_{n}=2^{n-1} \Im z$ for all $n \in \mathbb{N} \cup\{0\}$. Let $J_{n}=\left[\Re z-s_{n}, \Re z+s_{n}\right]$ and $G_{0}=J_{0}$, $G_{n}=J_{n} \backslash J_{n-1}$. We get

$$
\begin{aligned}
& |f(z)|^{p} \leq \sum_{n=0}^{\infty} \int_{G_{n}} P_{\gamma}(z-t, s)|f(s+i t)|^{p} d s \\
& \leq \sum_{n=1}^{\infty}\left[P_{\gamma}\left(z-t, \Re z+s_{n-1}\right)+P_{\gamma}\left(z-t, \Re z-s_{n-1}\right)\right] \int_{G_{n}}|f(s+i t)|^{p} d s \\
& \quad+P_{\gamma}(z-t, \Re z) \int_{G_{0}}|f(s+i t)|^{p} d s \\
& \leq \sum_{n=0}^{\infty} \frac{1}{\Im z} \frac{1}{2^{n \gamma}} \int_{G_{n}}|f(s+i t)|^{p} d s .
\end{aligned}
$$

Thus,

$$
|f(z)|^{p}(\Im z) \int_{0}^{\frac{\Im}{2}} d \omega(t) \lesssim \sum_{n=0}^{\infty} \frac{1}{2^{n \gamma}} \int_{0}^{\frac{\Im}{2}} \int_{G_{n}}|f(s+i t)|^{p} d s d \omega(t)
$$

It follows from (2.1) that

$$
\begin{aligned}
|f(z)|^{p} & \lesssim \sum_{n=0}^{\infty} \frac{1}{2^{n(\gamma-\beta-1)}} \frac{\int_{0}^{s_{n+1}} \int_{G_{n}}|f(s+i t)|^{p} d s d \omega(t)}{2^{n} \Im z \int_{0}^{s_{n+1}} d \omega(t)} \\
& \leq M_{\omega}\left(f^{p}\right)(z)\left(\sum_{n=0}^{\infty} \frac{1}{2^{n(\gamma-\beta-1)}}\right) \\
& \lesssim M_{\omega}\left(f^{p}\right)(z) .
\end{aligned}
$$

The proof is completed.

We are now ready to prove the compact Carleson embedding theorem.

Proof of Theorem 1.2 For the implication $(1) \Rightarrow(3)$, assume that $\mu$ is a compact $p$ Carleson measure for $A_{\omega}^{p}\left(\Pi^{+}\right)$. Consider the test functions

$$
f_{z, p}(w)=\left(\frac{\Im z}{w-\bar{z}}\right)^{\frac{\eta}{p}} \frac{1}{(\omega[Q(z)])^{\frac{1}{p}}}
$$

where $\eta>\lambda_{0}+1$ is sufficiently large. Then $\left\{f_{z, p}\right\}$ is a bounded sequence in $A_{\omega}^{p}\left(\Pi^{+}\right)$ by Lemma 2.1. For $\varepsilon>0$, put

$$
K_{\varepsilon}=\left\{w \in \Pi^{+}:|w-\varepsilon i| \leq \frac{1}{\varepsilon} \text { and } \Im w \geq \varepsilon\right\}
$$


and

$$
G_{\varepsilon}=\Pi^{+} \backslash K_{\varepsilon}
$$

Since $\mu$ is a compact $p$-Carleson measure for $A_{\omega}^{p}\left(\Pi^{+}\right)$, the closure $\overline{\left\{f_{z, p}: z \in \Pi^{+}\right\}}$ is compact in $L^{p}(\mu)$. Thus

$$
\lim _{\varepsilon \rightarrow 0^{+}} \int_{\Pi^{+}}\left|\chi_{G_{\varepsilon}}(w) f_{z, p}(w)\right|^{p} d \mu(w)=0
$$

uniformly in $z$. Noting that $\left\{f_{z, p}\right\}$ is a bounded sequence in $A_{\omega}^{p}\left(\Pi^{+}\right)$and converges uniformly on compact sets of $\Pi^{+}$to 0 as $z \rightarrow \partial \widehat{\Pi^{+}}$, we have from (3.4) that

$$
\lim _{z \rightarrow \partial \widehat{\Pi}^{+}}\left\|f_{z, p}\right\|_{L^{p}(\mu)}=0 .
$$

Thus,

$$
\lim _{z \rightarrow \partial \widehat{\Pi^{+}}} \frac{1}{\omega[Q(z)]} \int_{\Pi^{+}}\left(\frac{\Im z}{|w-\bar{z}|}\right)^{\eta} d \mu(w)=0 .
$$

The implication $(3) \Rightarrow(2)$ is immediate. For the implication $(2) \Rightarrow(1)$, suppose that

$$
\lim _{z \rightarrow \partial{\widehat{\Pi^{+}}}^{+}} \frac{\mu[Q(z)]}{\omega[Q(z)]}=0 .
$$

That is, for any $\varepsilon>0$, there is a compact set $K \subset \Pi^{+}$such that

$$
\sup _{z \in \Pi^{+} \backslash K} \frac{\mu[Q(z)]}{\omega[Q(z)]}<\varepsilon .
$$

Let $r_{1}=\sup _{z \in K}|\Re z|, r_{2}=\sup _{z \in K} \Im z$, and $s=\inf _{z \in K} \Im \Im_{z}$. Denote $K_{Q}=\left\{z \in \Pi^{+}\right.$: $\left.-r_{1} \leq \Re z \leq r_{1}, \frac{s}{3} \leq \Im z \leq r_{2}\right\}$. Then,

$$
\sup _{z \in \Pi^{+} \backslash K_{Q}} \frac{\mu[Q(z)]}{\omega[Q(z)]} \leq \sup _{z \in \Pi^{+} \backslash K} \frac{\mu[Q(z)]}{\omega[Q(z)]}<\varepsilon .
$$

Any bounded sequence $\left\{f_{n}\right\}$ in $A_{\omega}^{p}\left(\Pi^{+}\right)$is uniformly bounded on compact subsets of $\Pi^{+}$by Lemma 3.4. A well-known application of Montel's theorem together with Fatou's lemma gives us a subsequence $\left\{f_{n_{k}}\right\}$ that converges uniformly on compact sets of $\Pi^{+}$to a function $f \in A_{\omega}^{p}\left(\Pi^{+}\right)$. Set

$$
d \mu_{G}(z)=\chi_{\Pi^{+} \backslash K_{Q}}(z) d \mu(z) .
$$

Denote $I_{K_{Q}}=\left\{x \in \mathbb{R}:-r_{1} \leq x \leq r_{1}\right\}$. If $z \in\left\{K_{Q}: I_{z} \cap\left(\mathbb{R} \backslash I_{K_{Q}}\right)=\emptyset\right\}$, we

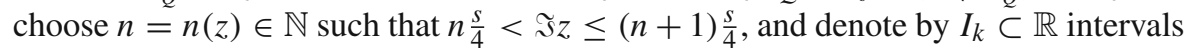


satisfying $\left|I_{k}\right|=\frac{s}{2}$ for all $k=1, \ldots, n$ and $I_{z} \subset \cup_{k=1}^{n+1} I_{k}$. By (3.5) and the doubling property of $\omega$,

$$
\begin{aligned}
\mu_{G}[Q(z)] & \leq \sum_{k=1}^{n+1} \mu\left[Q_{I_{k}}\right] \leq \varepsilon \sum_{k=1}^{n+1} \omega\left[Q_{I_{k}}\right] \\
& =\varepsilon(n+1) \frac{s}{2} \int_{0}^{\frac{s}{2}} d \omega(y) \leq 4 C \varepsilon n \frac{s}{4} \int_{0}^{\frac{s}{4}} d \omega(y) \\
& \leq 4 C \varepsilon \omega[Q(z)] .
\end{aligned}
$$

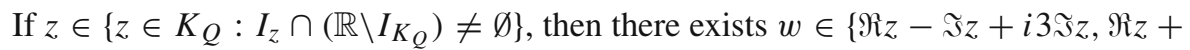
$\Im z+i 3 \Im z\} \backslash K_{Q}$. By (3.5) and Lemma 2.1, there exists a constant $C^{\prime}=C^{\prime}(\omega)>0$ such that

$$
\frac{\mu_{G}[Q(z)]}{\omega[Q(z)]} \leq C^{\prime} \frac{\mu_{G}[Q(w)]}{\omega[Q(w)]} \leq C^{\prime} \varepsilon
$$

Due to (3.5),

$$
\frac{\mu_{G}[Q(z)]}{\omega[Q(z)]}<\varepsilon, z \in \Pi^{+} \backslash K_{Q}
$$

Consequently,

$$
\sup _{z \in \Pi^{+}} \frac{\mu_{G}[Q(z)]}{\omega[Q(z)]}<2\left(4 C+C^{\prime}+1\right) \varepsilon
$$

Theorem 1.1 implies

$$
\begin{aligned}
& \int_{\Pi^{+}}\left|f_{n_{k}}(z)-f(z)\right|^{p} d \mu(z) \\
& \quad=\int_{K_{Q}}\left|f_{n_{k}}(z)-f(z)\right|^{p} d \mu(z)+\int_{\Pi^{+}}\left|f_{n_{k}}(z)-f(z)\right|^{p} d \mu_{G}(z) \\
& \quad \lesssim \int_{K_{Q}}\left|f_{n_{k}}(z)-f(z)\right|^{p} d \mu(z)+\sup _{z \in \Pi^{+}} \frac{\mu_{G}[Q(z)]}{\omega[Q(z)]}
\end{aligned}
$$

Since $\left\{f_{n_{k}}\right\}$ converges uniformly on compact sets of $\Pi^{+}$and $\mu$ is finite on $K$,

$$
\limsup _{k \rightarrow \infty} \int_{\Pi^{+}}\left|f_{n_{k}}(z)-f(z)\right|^{p} d \mu(z) \lesssim \sup _{z \in \Pi^{+}} \frac{\mu_{G}[Q(z)]}{\omega[Q(z)]}<2\left(4 C+C^{\prime}+1\right) \varepsilon
$$

Since $\varepsilon$ is arbitrary, we see $f_{n_{k}} \rightarrow f$ in $L^{p}(\mu)$, that is, $\mu$ is a compact $p$-Carleson measure for $A_{\omega}^{p}\left(\Pi^{+}\right)$. 


\section{Area Operators}

In this section we prove Theorem 1.3 by adapting the unit disk case [22]. To this end, a couple of lemmas are needed. We first define the following dyadic grids and the corresponding maximal operators that have recently been used in harmonic analysis, see $[22,24]$ and the references therein.

$$
\mathcal{D}^{\theta}:=\left\{\left[2^{j}\left(m+(-1)^{j} \theta\right), 2^{j}\left(1+m+(-1)^{j} \theta\right)\right), \quad m, j \in \mathbb{Z}\right\}, \quad \theta \in\left\{0, \frac{1}{3}\right\} .
$$

Lemma 4.1 [24, Lemma 3.1] If I is an interval in $\mathbb{R}$, then there exists an interval $K \in \mathcal{D}^{\theta}$ for some $\theta \in\left\{0, \frac{1}{3}\right\}$ such that $I \subset K$ and $|K| \leq 8|I|$.

For a positive Borel measure $\mu$ and a dyadic grid $\mathcal{D}^{\theta}$, we define

$$
M_{\mu, \mathcal{D}^{\theta}}(f)(z)=\sup _{I \in \mathcal{D}^{\theta}: z \in Q_{I}} \frac{1}{\mu\left[Q_{I}\right]} \int_{Q_{I}}|f(w)| d \mu(w)
$$

These dyadic maximal operators are "essentially insensitive to the underlying measure" [12]—-we have the following variant of this fundamental fact:

Lemma 4.2 Let $1<p<\infty$. Let $\mu$, v be positive Borel measures. For a dyadic grid $\mathcal{D}^{\theta}$ with $\theta \in\left\{0, \frac{1}{3}\right\}$, if

$$
\sup _{I \in \mathcal{D}^{\theta}} \frac{v\left[Q_{I}\right]}{\mu\left[Q_{I}\right]}<\infty
$$

then $M_{\mu, \mathcal{D}^{\theta}}: L^{p}(\mu) \rightarrow L^{p}(v)$ is bounded. Moreover,

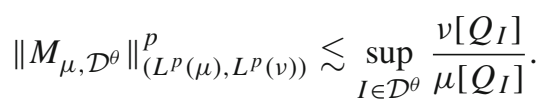

Proof We will use the Marcinkiewicz interpolation theorem, and therefore it suffices to prove weak type- $(1,1)$ inequality ( $L^{\infty}$ estimate being obvious). Let $s>0, f \in L^{1}(\mu)$, and

$$
d_{f}(s)=\left\{z \in \Pi^{+}: M_{\mu, \mathcal{D}^{\theta}}(f)(z)>s\right\}
$$

If $d_{f} f(s)=\emptyset$, then there is nothing to prove. We now suppose that $d_{f}(s) \neq \emptyset$. For $T>0$, define the sets

$$
A_{s}^{T}=\left\{Q_{I}: I \in \mathcal{D}^{\theta},|I|<T, \int_{Q_{I}}|f| d \mu \geq s \mu\left[Q_{I}\right]\right\} .
$$


Let $A_{s}^{T, \max }$ be the subfamily of $A_{s}^{T}$ consisting of the maximal $Q_{I}$ under inclusion. Since $|I|<T$, finding such a family of maximal elements is possible. It follows that $A_{S}^{T, \text { max }}$ is a covering of

$$
d_{f}(T, s)=\left\{z \in \Pi^{+}: \sup _{I \in \mathcal{D}^{\theta}: z \in Q_{I},|I|<T} \frac{1}{\mu\left[Q_{I}\right]} \int_{Q_{I}}|f| d \mu>s\right\}
$$

and the squares $Q_{I}$ from $A_{s}^{T, \max }$ are mutually disjoint. Then clearly each $z \in d_{f}(T, s)$ is contained at most one square in $A_{S}^{T, \text { max }}$. Therefore,

$$
\begin{aligned}
\nu\left[d_{f}(T, s)\right] & \leq \sum_{Q_{I} \in A_{s}^{T, \text { max }}} v\left[Q_{I}\right] \leq\left(\sup _{I \in \mathcal{D}^{\theta}} \frac{\nu\left[Q_{I}\right]}{\mu\left[Q_{I}\right]}\right) \sum_{Q_{I} \in A_{s}^{T, \max }} \mu\left[Q_{I}\right] \\
& \leq\left(\sup _{I \in \mathcal{D}^{\theta}} \frac{\nu\left[Q_{I}\right]}{\mu\left[Q_{I}\right]}\right) \frac{1}{s} \sum_{Q_{I} \in A_{s}^{T, \max }} \int_{Q_{I}}|f| d \mu \\
& \leq\left(\sup _{I \in \mathcal{D}^{\theta}} \frac{\nu\left[Q_{I}\right]}{\mu\left[Q_{I}\right]}\right) \frac{1}{s}\|f\|_{L^{1}(\mu)} .
\end{aligned}
$$

When $T \rightarrow \infty$, the sets $d_{f}(T, s)$ expand and $d_{f}(s)=\cup_{T>0} d_{f}(T, s)$. So

$$
v\left[d_{f}(s)\right]=\lim _{T \rightarrow \infty} v\left[d_{f}(T, s)\right] \leq\left(\sup _{I \in \mathcal{D}^{\theta}} \frac{v\left[Q_{I}\right]}{\mu\left[Q_{I}\right]}\right) \frac{1}{s}\|f\|_{L^{1}(\mu)}
$$

which completes the proof.

We are now ready to prove Theorem 1.3.

Proof of Theorem 1.3 We divide the proof into three cases: (i) $p=s$, (ii) $p>s$, and (iii) $p<s$.

Case (i): It follows directly from Fubini's theorem that

$$
\begin{aligned}
\left\|A_{s}^{\mu} f\right\|_{L^{p}(d(\omega \otimes m))}^{p} & =\int_{\Pi^{+}} \int_{\Lambda_{T}(z)}|f(w)|^{p} d \mu(w) d(\omega \otimes m)(z) \\
& =\int_{\Pi^{+}}|f(w)|^{p} d \mu_{\omega}(w) .
\end{aligned}
$$

Hence $A_{s}^{\mu}$ is bounded if and only if $\mu_{\omega}$ is a $p$-Carleson measure for $A_{\omega}^{p}\left(\Pi^{+}\right)$.

Case (ii): Assume that $A_{s}^{\mu}: A_{\omega}^{p}\left(\Pi^{+}\right) \rightarrow L^{p}(d(\omega \otimes m))$ is bounded. Considering the test functions $F_{z, p}$ defined in Lemma 2.1, we have 


$$
\begin{aligned}
\mu_{\omega}[Q(z)] & \approx \int_{Q(z)}\left|F_{z, p}(w)\right|^{s} d \mu_{\omega}(w) \\
& \approx \int_{Q(z)}\left|F_{z, p}(w)\right|^{s} \int_{T(w)}\left|F_{z,(p / s)^{\prime}}(u)\right| d(\omega \otimes m)(u) d \mu(w) .
\end{aligned}
$$

Using Fubini's theorem, we obtain that

$$
\mu_{\omega}[Q(z)] \lesssim \int_{\Pi^{+}}\left|F_{z,(p / s)^{\prime}}(u)\right| \int_{\Lambda_{T}(u)}\left|F_{z, p}(w)\right|^{s} d \mu(w) d(\omega \otimes m)(u) .
$$

Using Hölder's inequality and Lemma 2.1 yields that

$$
\mu_{\omega}[Q(z)] \lesssim\left\|A_{s}^{\mu}\right\|^{s} \omega[Q(z)]
$$

which implies that $d \mu_{\omega}(z)=\omega[T(z)] d \mu(z)$ is a $p$-Carleson measure for $A_{\omega}^{p}\left(\Pi^{+}\right)$by Theorem 1.1.

Conversely, assume that $d \mu_{\omega}(z)=\omega[T(z)] d \mu(z)$ is a $p$-Carleson measure for $A_{\omega}^{p}\left(\Pi^{+}\right)$. For brevity, we denote $M_{(\omega \otimes m), \mathcal{D}^{\theta}}$ by $M_{\omega, \mathcal{D}^{\theta}}$, where $\theta \in\{0,1 / 3\}$. We also write $\mathcal{B}\left((p / s)^{\prime}\right)$ for the unit ball of $L^{(p / s)^{\prime}}(d(\omega \otimes m))$.

Due to

$$
\begin{aligned}
& \left\|A_{s}^{\mu} f\right\|_{L^{p}(d(\omega \otimes m))}^{s} \\
& =\sup _{h \in \mathcal{B}\left((p / s)^{\prime}\right)} \int_{\Pi^{+}}|h(z)| \int_{\Lambda_{T}(z)}|f(w)|^{s} \frac{d \mu_{\omega}(w)}{\omega[T(w)]} d(\omega \otimes m)(z),
\end{aligned}
$$

it follows from Lemma 4.1, and Lemma 2.1 that

$$
\begin{aligned}
& \left\|A_{s}^{\mu} f\right\|_{L^{p}(d(\omega \otimes m))}^{s} \\
& \quad=\sup _{h \in \mathcal{B}\left((p / s)^{\prime}\right)} \int_{\Pi^{+}}|f(w)|^{s} \frac{1}{\omega[T(w)]} \int_{T(w)}|h(z)| d(\omega \otimes m)(z) d \mu_{\omega}(w) \\
& \quad \lesssim \sup _{h \in \mathcal{B}\left((p / s)^{\prime}\right)} \int_{\Pi^{+}}|f(w)|^{s}\left(M_{\omega, \mathcal{D}^{0}}(h)(w)+M_{\omega, \mathcal{D}^{1 / 3}}(h)(w)\right) d \mu_{\omega}(w) .
\end{aligned}
$$

Then, by Hölder's inequality, Lemma 4.2, and Theorem 1.1, we have

$$
\left\|A_{s}^{\mu} f\right\|_{L^{p}(d(\omega \otimes m))}^{s} \lesssim\left(\sup _{z \in \Pi^{+}} \frac{\mu_{\omega}[Q(z)]}{\omega[Q(z)]}\right)\|f\|_{A_{\omega}^{p}}^{s},
$$

that is, $A_{s}^{\mu}: A_{\omega}^{p}\left(\Pi^{+}\right) \rightarrow L^{p}(d(\omega \otimes m))$ is bounded.

Case (iii): Assume that $A_{s}^{\mu}: A_{\omega}^{p}\left(\Pi^{+}\right) \rightarrow L^{p}(d(\omega \otimes m))$ is bounded. For $\varepsilon>0$, put

$$
K_{\varepsilon}=\left\{w \in \Pi^{+}:|w-\varepsilon i| \leq \frac{1}{\varepsilon} \text { and } \Im w \geq \varepsilon\right\}
$$


and define $d \mu_{\varepsilon}(z)=\chi_{K_{\varepsilon}}(z) d \mu(z)$. Obviously, it holds that

$$
\left\|A_{s}^{\mu_{\varepsilon}}\right\|_{\left(A_{\omega}^{p}, L^{p}(d(\omega \otimes m))\right)} \leq\left\|A_{s}^{\mu}\right\|_{\left(A_{\omega}^{p}, L^{p}(d(\omega \otimes m))\right)} .
$$

Take $\alpha>\beta>1$ satisfying $\frac{\beta}{\alpha}=\frac{p}{s}$. It follows from Fubini's theorem that

$$
\begin{aligned}
\left(\mu_{\varepsilon}\right)_{\omega}[Q(z)] & =\int_{\Pi^{+}} \chi_{Q(z)}(w) d\left(\mu_{\varepsilon}\right)_{\omega}(w) \\
& \approx \int_{\Pi^{+}} \chi_{Q(z)}(w)\left|F_{z, p}(w)\right|^{s} \int_{T(w)} d(\omega \otimes m)(u) d \mu_{\varepsilon}(w) \\
& =\int_{\Pi^{+}}\left(\int_{\Lambda_{T}(u)} \chi_{Q(z)}(w)\left|F_{z, p}(w)\right|^{s} d \mu_{\varepsilon}(w)\right)^{\frac{1}{\alpha}+\frac{1}{\alpha^{\prime}}} d(\omega \otimes m)(u)
\end{aligned}
$$

By Hölder's inequality we have

$$
\begin{aligned}
&\left(\mu_{\varepsilon}\right)_{\omega}[Q(z)] \lesssim\left(\int_{\Pi^{+}}\left(\int_{\Lambda_{T}(u)}\left|F_{z, p}(w)\right|^{s} d \mu_{\varepsilon}(w)\right)^{\frac{\beta}{\alpha}} d(\omega \otimes m)(u)\right)^{\frac{1}{\beta}} \\
& \times\left(\int_{\Pi^{+}}\left(\int_{\Lambda_{T}(u)} \chi Q(z)(w) d \mu_{\varepsilon}(w)\right)^{\frac{\beta^{\prime}}{\alpha^{\prime}}} d(\omega \otimes m)(u)\right)^{\frac{1}{\beta^{\prime}}} \\
&=\left\|A_{S}^{\mu_{\varepsilon}} F_{z, p}\right\|_{L^{p}(d(\omega \otimes m))}^{\frac{p}{\beta}}\left\|A_{1}^{\mu_{\varepsilon}} \chi Q(z)\right\|_{L^{\frac{\beta^{\prime}}{\alpha^{\prime}}(d(\omega \otimes m))}}^{\frac{1}{\alpha^{\prime}}} .
\end{aligned}
$$

Observe that $\left(\frac{\beta^{\prime}}{\alpha^{\prime}}\right)^{\prime}=\frac{\beta(\alpha-1)}{\alpha-\beta}$, and write again $\mathcal{B}\left(\frac{\beta(\alpha-1)}{\alpha-\beta}\right)$ for the unit ball of $L^{\frac{\beta(\alpha-1)}{\alpha-\beta}}(d(\omega \otimes m))$. Applying Fubini's theorem, Lemma 4.1, and Lemma 2.1, we have by duality

$$
\begin{aligned}
& \left\|A_{1}^{\mu_{\varepsilon}} \chi_{Q(z)}\right\|{ }_{L^{\frac{\beta^{\prime}}{\alpha^{\prime}}}(d(\omega \otimes m))} \\
& =\sup _{h \in \mathcal{B}\left(\frac{\beta(\alpha-1)}{\alpha-\beta}\right)} \int_{\Pi^{+}}|h(u)| A_{1}^{\mu_{\varepsilon}} \chi_{Q(z)}(u) d(\omega \otimes m)(u) \\
& =\sup _{h \in \mathcal{B}\left(\frac{\beta(\alpha-1)}{\alpha-\beta}\right)} \int_{\Pi^{+}}|h(u)|\left(\int_{\Lambda_{T}(u)} \chi_{Q(z)}(w) d \mu_{\varepsilon}(w)\right) d(\omega \otimes m)(u) \\
& =\sup _{h \in \mathcal{B}\left(\frac{\beta(\alpha-1)}{\alpha-\beta}\right)} \int_{\Pi^{+}} \chi_{Q(z)}(w) \frac{1}{\omega[T(w)]} \int_{T(w)}|h(u)| d(\omega \otimes m)(u) d\left(\mu_{\varepsilon}\right)_{\omega}(w) \\
& \lesssim \sup _{h \in \mathcal{B}\left(\frac{\beta(\alpha-1)}{\alpha-\beta}\right)} \int_{\Pi^{+}} \chi Q(z)(w)\left(M_{\omega, \mathcal{D}^{0}}(h)(w)+M_{\omega, \mathcal{D}^{1 / 3}}(h)(w)\right) d\left(\mu_{\varepsilon}\right)_{\omega}(w) .
\end{aligned}
$$


Using Hölder's inequality and Lemma 4.2, we have

$$
\left\|A_{1}^{\mu_{\varepsilon}} \chi_{Q(z)}\right\|_{L^{\frac{\beta^{\prime}}{\alpha^{\prime}}(d(\omega \otimes m))}} \lesssim\left(\left(\mu_{\varepsilon}\right)_{\omega}[Q(z)]\right)^{\frac{\alpha^{\prime}}{\beta^{\prime}}}\left(\sup _{w \in \Pi^{+}} \frac{\left(\mu_{\varepsilon}\right)_{\omega}[Q(w) \cap Q(z)]}{\omega[Q(w)]}\right)^{1-\frac{\alpha^{\prime}}{\beta^{\prime}}} .
$$

This estimate together with (4.1) and Lemma 2.1 gives us

$$
\begin{aligned}
& \left(\left(\mu_{\varepsilon}\right)_{\omega}[Q(z)]\right)^{\frac{1}{\beta}}(\omega[Q(z)])^{-\frac{1}{\beta}} \\
& \quad \lesssim\left\|A_{s}^{\mu_{\varepsilon}}\right\|_{\left(A_{\omega}^{p}, L^{p}(d(\omega \otimes m))\right)}^{\frac{p}{\beta}}\left(\sup _{w \in \Pi^{+}} \frac{\left(\mu_{\varepsilon}\right)_{\omega}[Q(w) \cap Q(z)]}{\omega[Q(w)]}\right)^{\frac{1}{\alpha^{\prime}}-\frac{1}{\beta^{\prime}}} .
\end{aligned}
$$

Therefore,

$$
\sup _{z \in \Pi^{+}, \varepsilon>0} \frac{\left(\mu_{\varepsilon}\right)_{\omega}[Q(z)]}{\omega[Q(z)]} \lesssim\left\|A_{s}^{\mu}\right\|_{\left(A_{\omega}^{p}, L^{p}(d(\omega \otimes m))\right)}^{s} .
$$

It follows from Fatou's lemma and Theorem 1.1 that $\mu_{\omega}$ is a $p$-Carleson measure for $A_{\omega}^{p}\left(\Pi^{+}\right)$and

$$
\sup _{z \in \Pi^{+}} \frac{\mu_{\omega}[Q(z)]}{\omega[Q(z)]} \lesssim\left\|A_{s}^{\mu}\right\|_{\left(A_{\omega}^{p}, L^{p}(d(\omega \otimes m))\right)}^{s}
$$

Conversely, assume that $d \mu_{\omega}(z)=\omega[T(z)] d \mu(z)$ is a $p$-Carleson measure for $A_{\omega}^{p}\left(\Pi^{+}\right)$. Applying Hölder's inequality we have

$$
\begin{aligned}
\left\|A_{s}^{\mu} f\right\|_{L^{p}(d(\omega \otimes m))}^{p} & \int_{\Pi^{+}}\left(\int_{\Lambda_{T}(z)}|f(w)|^{s} \frac{d \mu_{\omega}(w)}{\omega[T(w)]}\right)^{\frac{p}{s}} d(\omega \otimes m)(z) \\
\leq & \int_{\Pi^{+}}\left(N_{T} f(z)\right)^{\frac{p(s-p)}{s}}\left(\int_{\Lambda_{T}(z)}|f(w)|^{p} \frac{d \mu_{\omega}(w)}{\omega[T(w)]}\right)^{\frac{p}{s}} d(\omega \otimes m)(z) \\
\leq & \left(\int_{\Pi^{+}}\left(N_{T} f(z)\right)^{p} d(\omega \otimes m)(z)\right)^{1-\frac{p}{s}} \\
& \times\left(\int_{\Pi^{+}} \int_{\Lambda_{T}(z)}|f(w)|^{p} \frac{d \mu_{\omega}(w)}{\omega[T(w)]} d(\omega \otimes m)(z)\right)^{\frac{p}{s}} .
\end{aligned}
$$

Finally, Fubini's theorem, Theorem 1.1, and Lemma 3.2 yield

$$
\begin{aligned}
\left\|A_{s}^{\mu} f\right\|_{L^{p}(d(\omega \otimes m))}^{p} & \lesssim\left\|N_{T} f\right\|_{L^{p}(d(\omega \otimes m))}^{\frac{p(s-p)}{s}}\|f\|_{L^{p}\left(\mu_{\omega}\right)}^{\frac{p^{2}}{s}} \\
& \lesssim\left(\sup _{z \in \Pi^{+}} \frac{\mu_{\omega}[Q(z)]}{\omega[Q(z)]}\right)^{\frac{p}{s}}\|f\|_{A_{\omega}^{p}}^{p},
\end{aligned}
$$

which completes the desired proof. 
Acknowledgements The authors thank the referees who provided numerous valuable comments that improved the overall presentation of the paper.

Funding Open access funding provided by Umea University.

Data availability Data sharing not applicable to this article as no datasets were generated or analysed during the current study: the article describes entirely theoretical research.

Open Access This article is licensed under a Creative Commons Attribution 4.0 International License, which permits use, sharing, adaptation, distribution and reproduction in any medium or format, as long as you give appropriate credit to the original author(s) and the source, provide a link to the Creative Commons licence, and indicate if changes were made. The images or other third party material in this article are included in the article's Creative Commons licence, unless indicated otherwise in a credit line to the material. If material is not included in the article's Creative Commons licence and your intended use is not permitted by statutory regulation or exceeds the permitted use, you will need to obtain permission directly from the copyright holder. To view a copy of this licence, visit http://creativecommons.org/licenses/by/4.0/.

\section{References}

1. Arsenović, M.: Embedding derivatives of $M$-harmonic functions into $L^{p}$ spaces. Rocky Mt. J. Math. 29, 61-67 (1999)

2. Carleson, L.: An interpolation problem for bounded analytic functions. Am. J. Math. 80, 921-930 (1958)

3. Carleson, L.: Interpolation by bounded analytic functions and the corona problem. Ann. Math. 76, 547-559 (1962)

4. Cohn, W.: Generalized area operators on Hardy spaces. J. Math. Anal. Appl. 216, 112-121 (1997)

5. Cohn, W., Verbitsky, I.: Factorization of tent spaces and Hankel operators. J. Funct. Anal. 175, 308-329 (2000)

6. Coifman, R., Meyer, Y., Stein, E.: Some new function spaces and their applications to Harmonic analysis. J. Funct. Anal. 62, 304-335 (1985)

7. Duren, P.: Theory of $H^{p}$ Spaces. Dover, New York (2000)

8. Garnett, J.: Bounded Analytic Functions. Academic Press, New York (1981)

9. Gong, M., Lou, Z., Wu, Z.: Area operators from $H^{p}$ spaces to $L^{q}$ spaces. Sci. China Math. 53, 357-366 (2010)

10. Harper, Z.: Boundedness of convolution operators and input-output maps between weighted spaces. Complex Anal. Oper. Theory 3(1), 113-146 (2009)

11. Hörmander, L.: $L^{p}$ estimates for (pluri-)subharmonic functions. Math. Scand. 20, 65-78 (1967)

12. Hytönen, T.: Dyadic Analysis and Weights. Lecture Notes. University of Helsinki, Helsinki (2017)

13. Jacob, B., Partington, J., Pott, S.: On Laplace-Carleson embedding theorems. J. Funct. Anal. 264, 783-814 (2013)

14. Kucik, A.: Spaces of analytic functions on the complex half-plane. Doctoral Thesis, University of Leeds (2017)

15. Luecking, D.: Embedding derivatives of Hardy spaces into Lebesgue spaces. Proc. Lond. Math. Soc. 63, 595-619 (1991)

16. Pang, C., Perälä, A., Wang, M., Guo, X.: Maximal estimate and integral operators in Bergman spaces with doubling measure. Proc. Am. Math. Soc. (to appear)

17. Pau, J., Peláez, J.Á.: Embedding theorems and integration operators on Bergman spaces with rapidly decreasing weights. J. Funct. Anal. 259, 2727-2756 (2010)

18. Peláez, J. Á.: Small weighted Bergman spaces. In: Proceedings of the Summer School in Complex and Harmonic Analysis, and Related Topics (2016)

19. Peláez, J.Á., Rättyä, J.: Weighted Bergman Spaces Induced by Rapidly Increasing Weights. Memoirs of the American Mathematical Society, No. 1066, vol. 227. American Mathematical Society, Providence (2014)

20. Peláez, J.Á., Rättyä, J.: Embedding theorems for Bergman spaces via harmonic analysis. Math. Ann. 362, 205-239 (2015) 
21. Peláez, J. Á., Rättyä, J.: Bergman projection induced by radial weight. Preprint (2019)

22. Peláez, J.Á., Rättyä, J., Sierra, K.: Embedding Bergman spaces into tent spaces. Math. Z. 281, 12151237 (2015)

23. Peloso, M.M., Salvatori, M.: On some spaces of holomorphic functions of exponential growth on a half-plane. Concr. Oper. 3(1), 52-67 (2016)

24. Pott, S., Reguera, M.C.: Sharp Bekollé estimate for the Bergman projection. J. Funct. Anal. 265, 3233-3244 (2013)

25. Ramey, W., Yi, H.: Harmonic Bergman functions on half-spaces. Trans. Am. Math. Soc. 348, 633-660 (1996)

26. Siskakis, A.: Weighted integrals of analytic functions. Acta Sci. Math. (Szeged) 66(3-4), 651-664 (2000)

27. Strömberg, J., Torchinsky, A.: Weights, sharp maximal functions and Hardy spaces. Bull. Am. Math. Soc. 3, 1053-1056 (1980)

28. Zhu, K.: Operator Theory in Function Spaces. Mathematical Surveys and Monographs, vol. 138, 2nd edn. American Mathematical Society, Providence (2007)

Publisher's Note Springer Nature remains neutral with regard to jurisdictional claims in published maps and institutional affiliations. 